\title{
Atelocollagen-delivered siRNA targeting the FABP5 gene as an experimental therapy for prostate cancer in mouse xenografts
}

\author{
SHIVA S. FOROOTAN, ZHENG Z. BAO, FARZAD S. FOROOTAN, LALEH KAMALIAN, \\ YU ZHANG, ALIX BEE, CHRISTOPHER S. FOSTER and YOUQIANG KE

\begin{abstract}
Division of Cellular Pathology and Molecular Genetics, School of Cancer Studies, University of Liverpool,
\end{abstract} \\ 6th Floor, Duncan Building, Daulby Street, Liverpool L69 3GA, UK
}

Received September 11, 2009; Accepted October 14, 2009

DOI: 10.3892/ijo_00000476

\begin{abstract}
The gene $F A B P 5$ encodes cutaneous fatty acid binding protein $(\mathrm{C}-\mathrm{FABP})$ that is up-regulated in prostate cancer where it acts as a putative oncogene. To test the hypothesis that siRNA to FABP5 delivered to the external environment of a prostate cancer would reduce the level of C-FABP in vivo, experiments were established whereby siRNA to FABP5 suspended in atelocollagen was injected around tumour masses produced by PC-3M cells in Balb/c nude mice and compared with the effect of non-specific scrambled siRNA in atelocollagen. At autopsy, the average size of tumours from the groups treated with 10 and $15 \mu \mathrm{M}$ siRNA in atelocollagen was significantly $(\mathrm{p}=0.02)$ reduced by more than 3 -fold, when compared to the controls. In contrast, when compared to the tumours produced by the group treated with scrambled siRNA, treatment with $10 \mu \mathrm{M}$ $F A B P 5$ siRNA in buffer and 1 or $5 \mu \mathrm{M}$ siRNA in atelocollagen did not produce significant differences. Although the dosage of $15 \mu \mathrm{M}$ siRNA produced a greater reduction in tumour sizes when compared with $10 \mu \mathrm{M}$, this difference was not significant $(\mathrm{p}=0.9)$. Immunohistochemistry and Western blotting revealed that the levels of C-FABP expression in tumours from mice treated with 10 and $15 \mu \mathrm{M}$ dosages were lower than those from the other groups. These data demonstrate that FABP5 siRNA delivered by atelocollagen to the external environment surrounding a tumour mass can effectively inhibit prostate cancer cell growth in nude mice when administered in a dose-dependent manner at concentrations of $>10 \mu \mathrm{M}$.
\end{abstract}

\section{Introduction}

Prostate cancers are characterised by clinical and biological heterogeneity with complex molecular and epidemiological

Correspondence to: Professor Y. Ke, Division of Cellular Pathology and Molecular Genetics, School of Cancer Studies, University of Liverpool, 6th Floor, Duncan Building, Daulby Street, Liverpool L69 3GA, UK

E-mail: yqk@liv.ac.uk

Key words: FABP5 siRNA, atelocollagen, prostate cancer, established tumours dimensions $(1,2)$. At the time of diagnosis, patients may have already developed metastatic disease, although this may not be clinically apparent. Effective treatment requires suppression of all tumour masses, in both primary and secondary sites. Thus, inhibition or reversal of malignant progression is essential to the effective clinical management of prostate cancer. Our recent work demonstrated that stably-expressing siRNA targeting FABP5 within cancer cells can effectively inhibit development of prostate cancer at the initiation stage (3). However, it is not clear whether or not siRNAs against $F A B P 5$ at the transcriptional level can effectively promote regression or inhibit further growth of existing tumours.

RNA interference (RNAi) has been considered to be a new tool for targeting genes responsible for the malignant progression of cancer cells. It has become a powerful technique capable of suppressing expression of individual genes with a high degree of specificity (4). Silencing RNA (siRNA) has also been considered as an experimental tool for exploring cellular pathways that lead to uncontrolled cell growth. Despite the great potential of the RNAi technique, application of siRNA molecules directly to malignant tumours as treatment drugs is still in the early stages of development. One of the main difficulties for utilizing siRNAs as treatment drugs is to keep the siRNA molecules stable so that they can remain long enough inside cancer cells to exert their biological effects. Thus, prevention of the siRNA molecules from rapid degradation is essential.

The rapid advancement of RNAi technology and the recent discovery of stable delivery systems, atelocollagen (5), has enabled the direct application of siRNAs to induce RNA interference in vivo $(6,7)$. This technical breakthrough has enabled appropriate conditions to study the direct effect of siRNA targeting FABP5 on already-existing cancer masses. Atelocollagen is a highly purified Type I collagen derived from calf dermis and treated with pepsin (Koken, Tokyo, Japan). It is low in immunogenicity because its telopeptides have been removed (8). Atellocollagen is soluble at low temperature $\left(<10^{\circ} \mathrm{C}\right)$ but solidified at body temperature (9). It has also been reported that the siRNA-atelocollagen complex is resistant to nucleases, potentially allowing long-term stability of short RNA molecules within the local environment surrounding cancer cells (8). Atelocollagen has been used clinically for a wide range of purposes, including wound healing, vessel prosthesis, as a bone and cartilage substitute, and for haemostasis (9), thus confirming its suitability for use in vivo. 
FABP5 was chosen as the target for siRNA since it is over-expressed in prostate cancer and the increased levels of C-FABP protein are considered to play an important role in the progression of prostate cancer cells $(3,10)$. Further, expression of C-FABP is a prognostic biomarker of patient outcome and suppression of FABP5 expression by stablytransfected siRNA has been shown to significantly inhibit the ability of prostate cancer cells to form tumours and to metastasize in nude mice (3). This study was performed to test the hypothesis that siRNA targeted to inhibit FABP5 and delivered in a stabilizing agent could be delivered directly but externally to a tumour mass as a therapeutic agent to treat prostate carcinomas already established in nude mice. To study this effect, siRNA to FABP5 was administered in atelocollagen around the tumours established in nude mice to assess the effectiveness and dose-response of stabilising this siRNA in the treatment of localised prostate cancer cells.

\section{Materials and methods}

Cell culture. The cell line used in this study was the highly malignant, metastatic prostate cancer cell line PC3-M (11) originally established from the most aggressive population of PC-3 cells (12). The cells were maintained as monolayer cultures in RPM-1640 (Life Technologies, Paisley, UK) and supplemented with $10 \%(\mathrm{v} / \mathrm{v})$ fetal calf serum (Labtech International, UK), penicillin $(100 \mathrm{U} / \mathrm{ml})$ and streptomycin $(100 \mathrm{mg} / \mathrm{ml})$.

Selection of siRNA sequences. To select the most effective silencing sequence, 5 siRNAs from the FABP5 cDNA were chosen through Ambion design tools (Fig. 1). Synthesized RNAs were purchased from Ambion (Austin, TX, USA) and each was dissolved in DNase- and RNase-free water to $100 \mu \mathrm{M}$. Scrambled RNA Neg1 was purchased from Ambion as a negative control. To identify the most effective target for FABP5 suppression, transient transfection of PC3-M cells with each of the FABP5 RNA sequences and scrambled RNA was performed using the siPORT ${ }^{\mathrm{TM}} \mathrm{NeoFX}^{\mathrm{TM}}$ transfection agent (Ambion). Briefly, adherent cells were tripsinized and diluted in normal growth medium to $1 \times 10^{5}$ cells per $\mathrm{ml}$. The siPORT NeoFX agent was diluted in OPTI-MEM medium and incubated for $10 \mathrm{~min}$ at room temperature. Thereafter, each siRNA was diluted separately in OPTI-MEM medium before the diluted RNA and diluted siPORT NeoFX agent were mixed, incubated at room temperature for $10 \mathrm{~min}$, and dispensed into culture plates. The PC3M cells were overlaid onto the RNA/siPORT NeoFX complex and incubated at $37^{\circ} \mathrm{C}$ in $5 \% \mathrm{CO}_{2}$ in air and $100 \%$ humidity for $72 \mathrm{~h}$. Western blotting was performed to measure the levels of 5 mRNA in cells transfected with different sequences. The siRNA sequence 25 (sense: 5'-AGCACAAUAACAAGAAAAUUU3', antisense: 5'-AUUUUCUUGUUAUUGUGCUUU-3') that produced the greatest reduction of FABP5 mRNA during transient transfection was identified as the most effective suppressor.

Western blotting. Expression of C-FABP protein in transiently transfected cells was detected by Western blot analysis using an ECL detection system (13-15). Proteins were separated by
SDS-PAGE in a $15 \%(\mathrm{w} / \mathrm{v})$ acrylamide gel, and transferred onto a nitrocellulose membrane (Hybond ECL, Amersham Pharmacia, Amersham, UK). The primary antibody against C-FABP (Iwaki Glass Corp., Japan) was diluted at 1:500 and incubated with the blot for $1 \mathrm{~h}$. After incubating with the horseradish peroxidase-conjugated swine anti-rabbit IgG (Dako) diluted at 1:1,000, detected protein bands were visualized by exposure to a Kodak XAR-5 film and quantified by measuring the intensity of the peak areas using an Alpha Imager 2,000 densitometer (Alpha Innotech, Cannock, UK). The antibody against the constitutively expressed actin protein was incubated with the same blot to correct possible loading errors.

In vivo studies. A total of 30 male Balb/c nude mice, 6-8 week old, were purchased commercially (Harlan, UK). For each mouse, $2 \times 10^{6}$ of PC3-M cells in $200 \mu 1$ PBS was injected subcutaneously into the flank region to establish tumours. Ateolocollagen-delivered siRNA treatment was administered to each mouse when the tumours had reached $50-60 \mathrm{~mm}^{3}$ in size. Tumours were measured every 3-4 days after they had become visualised. The volume of each tumour was calculated by the formula of LxWxHx0.5236 (16). All animal experiments were conducted according to UKCCCR guidelines under Home Office Project Licence PPL 40/2270 to Professor Y. Ke.

Experimental design. Mice were divided into six groups of five animals each. The groups were separately injected with the following: i) $10 \mu \mathrm{M}$ scrambled siRNA-atelocollagen; ii) $10 \mu \mathrm{M}$ C-FABP siRNA in the buffer supplied with the Atelogene kit; iii) $1 \mu \mathrm{M}$ C-FABP siRNA-atelocollagen; iv) $5 \mu \mathrm{M}$ C-FABP siRNA-atelocollagen; v) $10 \mu \mathrm{M}$ C-FABP siRNA-atelocollagen; and vi) $15 \mu \mathrm{M}$ C-FABP siRNA-atelocollagen. Injections were repeated at 7-day intervals and all animals were sacrificed at day 35 after the first injection. Animals were weighted and tumours measured in three dimensions every 3-4 days.

Atelocollagen. The stabilizing agent atelocollagen was purchased as siRNA delivery kit AteloGene (Koken, Tokyo, Japan) and stored at $4^{\circ} \mathrm{C}$. To prepare the siRNA-ateocollagen complex, equal volumes of siRNA solution and ateolocollagen were mixed by slow rotation in a round-bottom microtube at $4^{\circ} \mathrm{C}$ for $20 \mathrm{~min}$ at $4 \mathrm{rpm}$. For each injection, $200 \mu \mathrm{l}$ of this mixture was injected directly around each tumour mass growing in nude mice.

Immunohistochemistry and Western blotting of mousederived tumours. At autopsy, excised tumours were divided into two halves. One piece was flash-frozen. The other was fixed in neutral-buffered formaldehyde $(10 \% \mathrm{w} / \mathrm{v})$ and embedded in paraffin wax. From these, histological sections $(4 \mu \mathrm{m})$ were cut and mounted onto APES slides using standard routine procedures $(17,18)$. Tissue sections were stained with haematoxylin and eosin to assess tumour morphology. Serial tissue sections were stained immunohistochemically to detect C-FABP expression, as described previously $(14,19)$. The intensity of the immunohistochemical staining was classified into unstained (-), weak (+), moderate (++) and strongly positive $(+++)$. 


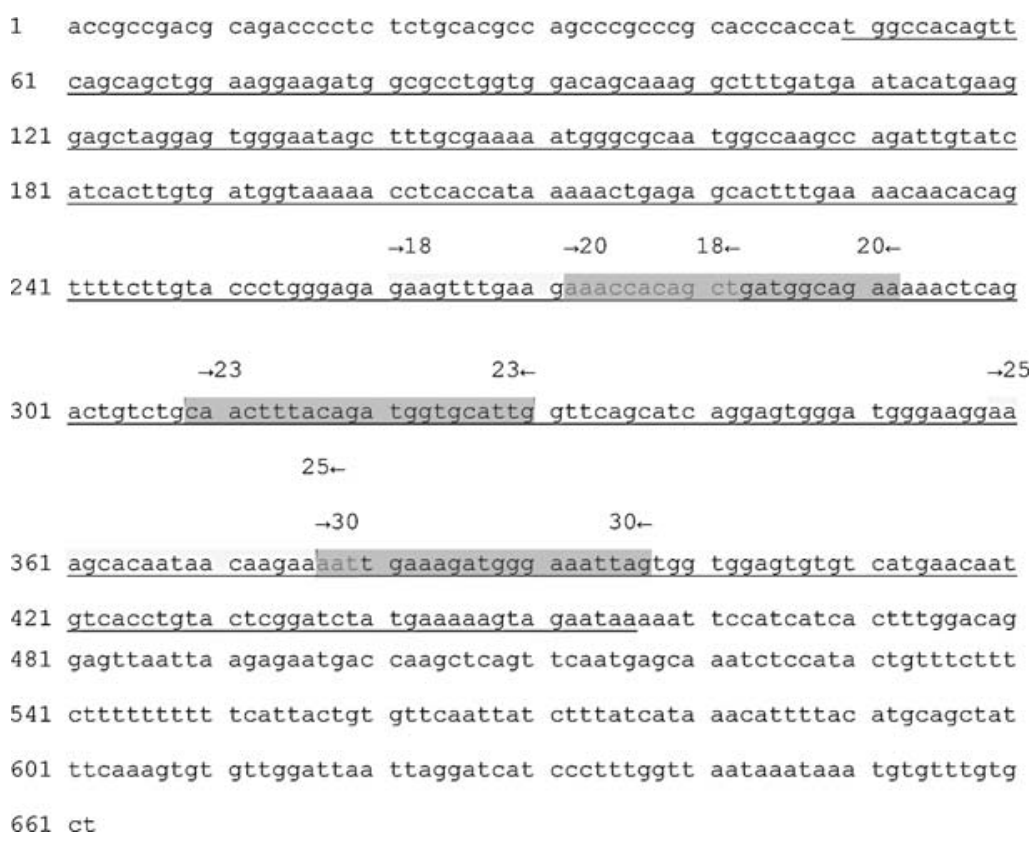

Figure 1. Selection of sequences for silencing FABP5 gene. Underlined area is the CDS region of the C-FABP cDNA and the highlighted 5 areas are the selected sequences for silencing RNA.

The other halves of the tumours that had been flashfrozen in liquid nitrogen and stored at $-80^{\circ} \mathrm{C}$, were thawed on the ice and lysed using of Cell lytic M cell lysate (Sigma) and proteinase inhibitors. Tissues were sonicated on full power for $3 \times 30 \mathrm{sec}$ on ice and centrifuged for $15 \mathrm{~min}$ at $4{ }^{\circ} \mathrm{C}$ and 13,000 rpm. Western blotting was performed as described (14).

Statistical analyses. Statistical Package for Social Sciences, Version 15 (SPSS Inc., Chicago, IL, USA) was used to analyse the experimental data. Student's t-test was used to compare the effect of different treatments. Mann-Whitney U test was used to assess the differences on weight or volume of tumours after different treatments. Differences amongst C-FABP levels detected in tumours after different treatments were assessed by $\chi^{2}$ analysis. Statistical significance was defined as $\mathrm{p}<0.05$.

\section{Results}

Suppression of FABP5 gene expression by different siRNAs. Western blot analysis of C-FABP expressed in PC3-M cells transiently transfected with different siRNA sequences is shown in Fig. 2. Sequence 25 exhibited greatest inhibition (Fig. 2a), although all other sequences also produced different levels of inhibition. Direct comparison of the efficiencies on FABP5 suppression produced by different siRNAs was performed by quantitative analysis (Fig. 2b). When the level of C-FABP mRNA expressed in PC3-M cells was set at 1, the level expressed in scrambled cells was 1.3 , which is similar to control. The levels of C-FABP mRNA in cells treated with five different siRNAs were greatly reduced to a range of 1.5-2.5, respectively; a 4.0-6.7-fold reduction. These data showed that sequences 18, 20, 23 and 30 produced around 2.5-fold reductions in C-FABP mRNA expression in PC3-M cells, whereas sequence 25 produced 6.7 - and 6.9 -fold

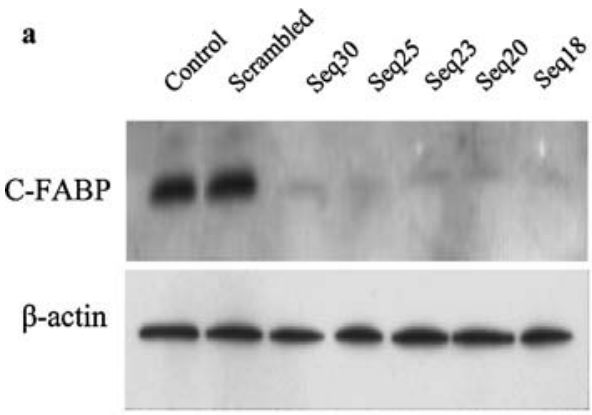

b

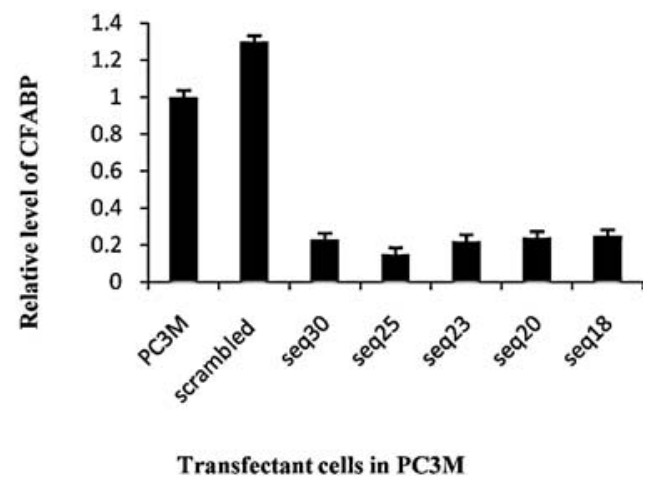

Figure 2. Levels of C-FABP expression in control and in each of the 5 different siRNA transfected cells. (a) Western blot analysis of C-FABP expression in PC3M cells, and cells transiently transfected with scrambled siRNA and 5 different FABP5 siRNAs (seq18, 20, 23, 25 and 30) respectively. B-actin antibody was also incubated with the same blot to normalized the possible loading discrepancies. (b) Relative levels of C-FABP expressed in PC3M cells, cells transiently transfected with scrambled siRNA and 5 different siRNAs (seq18, 20, 23, 25 and 30), respectively. The level of C-FABP expressed in PC3M was set at 1; C-FABP levels in each of the siRNA transfected cells were calculated by relating to the level expressed PC3M cells. The possible loading errors were corrected by normalizing values to $B$-actin, and the result is shown as the mean $( \pm \mathrm{SE})$ of three separate measurements. 
a

$10 \mu \mathrm{M}$ scrambled siRNA atelocollagen

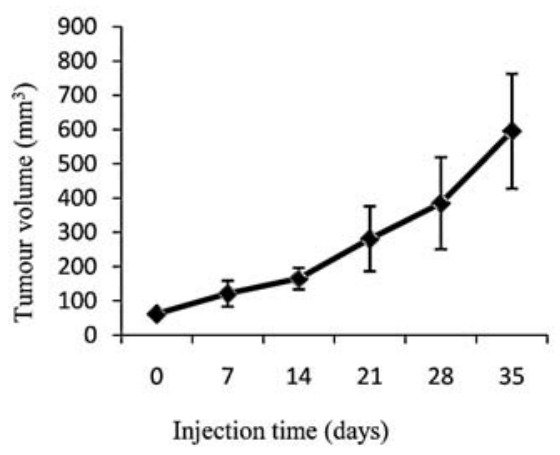

c

$5 \mu \mathrm{M} \mathrm{C}$-FABP siRNA -atelocollagen

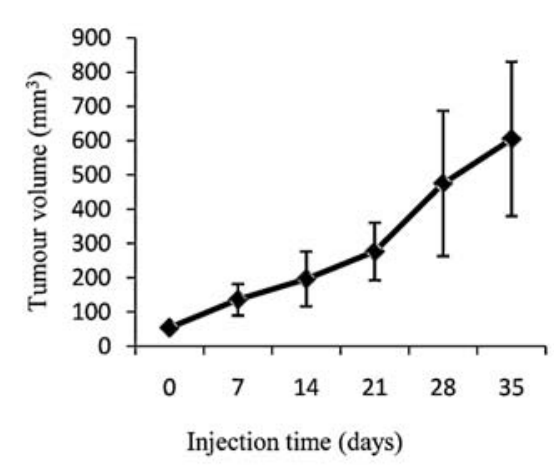

$\mathrm{e}$
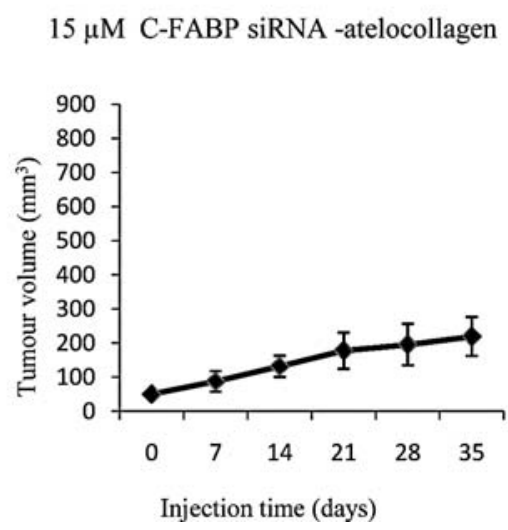

b

$1 \mu \mathrm{M}$ C-FABP siRNA - atelocollagen

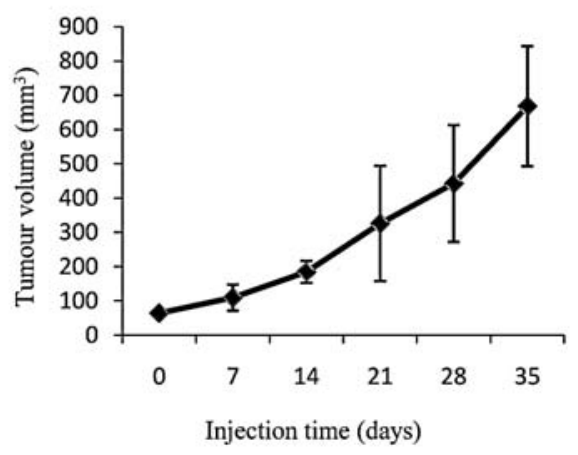

$\mathrm{d}$

$10 \mu \mathrm{M} \mathrm{C}$-FABP siRNA-atelocollagen

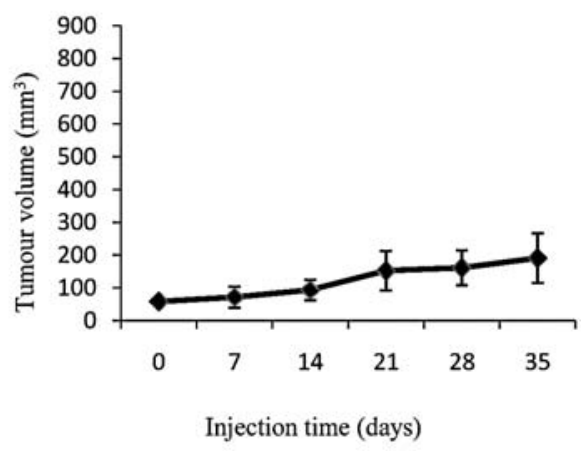

f

$10 \mu \mathrm{M} \mathrm{C}-\mathrm{FABP}$ siRNA in buffer

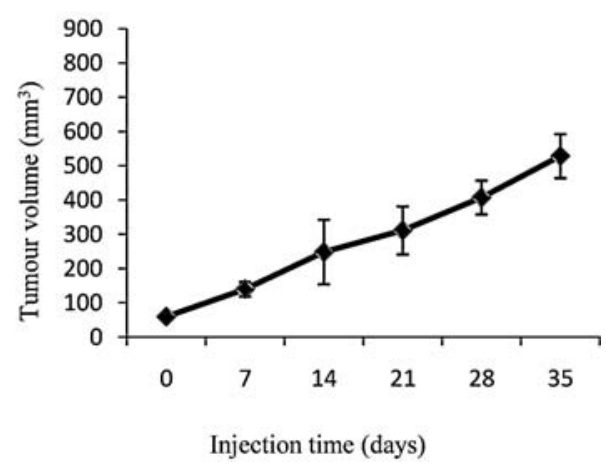

Figure 3. Different tumour-growing patterns in each group (5 mice) of animals treated without or with different doses of FABP5 RNAi-atelocollagen during the 35-day period.

reductions, respectively, in relation to the levels of C-FABP in untreated and scrambled cells. Thus, sequence 25 was the most effective suppressor of the target FABP5 gene.

Inhibition of tumour growth by FABP5 siRNA-atelocollagen complex. At 7-10 days after injecting the PC3-M cells, tumours were visible at the inoculation sites of all mice. When tumours reached 50-60 $\mathrm{mm}^{3}$, after 2-3 weeks, treatment with atelocollagen-containing scramble siRNA or FABP5 siRNA (sequence 25) was applied to the tumour mass to evaluate the therapeutic effect. While sizes of tumours from the two groups treated respectively with 10 and $15 \mu \mathrm{M}$ FABP5-siRNAatelocollagen exhibited a relatively small increases comparing with their sizes before the treatments, the sizes of tumours from all other groups appeared to be much larger at the end of the treatments comparing with their sizes before the treatments.

The size of tumours growing in each group was measured every 3 or 4 days following treatment. Detailed records on tumour growth reflected by their volumes for all six groups are shown in Fig. 3. Although the growth curves for most of the tumours in each group were similar, the scales of the tumour volumes were significantly lower in the two groups of mice treated with 10 and $15 \mu \mathrm{M} \mathrm{FABP5}$ siRNA-atelocollagen (Fig. 3d and e) than the control group (Fig. 3a) (Student's t-test $\mathrm{p}=0.002$ and $\mathrm{p}=0.001$ ). The increment in the 
a

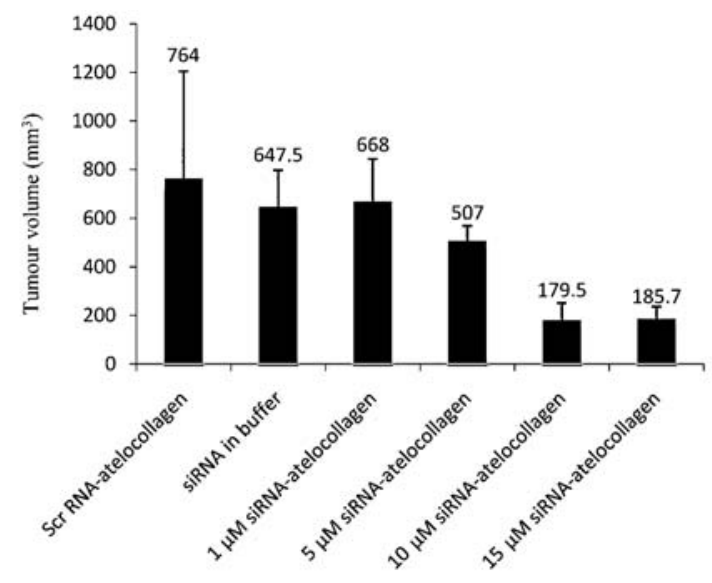

b

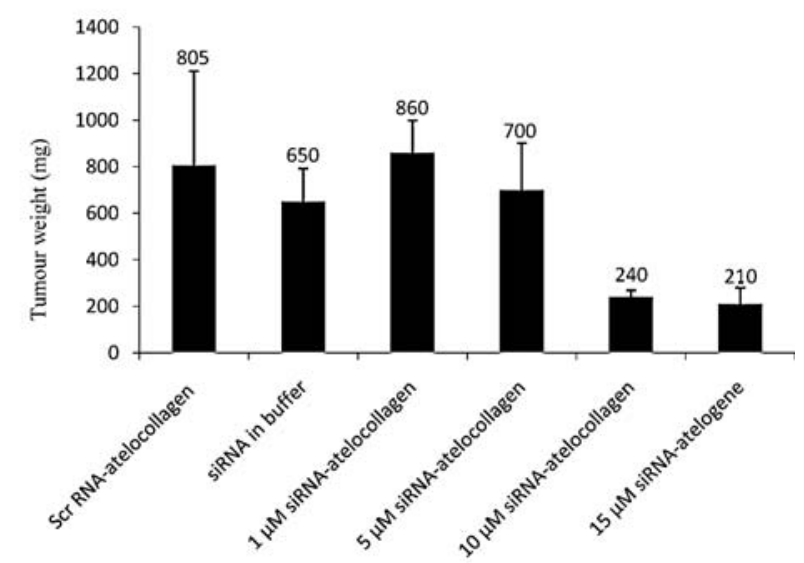

Figure 4. Average tumour volume (a) and weight (b) measured at the end of different treatments.

tumour volumes from the groups treated with $1 \mu \mathrm{M}$ (Student's t-test $\mathrm{p}=0.52$, Fig. 3b), $5 \mu \mathrm{M}$ (Student's t-test $\mathrm{p}=0.95$, Fig. 3c) $F A B P 5$ siRNA-atelocollagen and $10 \mu \mathrm{M} F A B P 5$ siRNA in buffer (Student's t-test $\mathrm{p}=0.2$, Fig. 3f) was not significantly different from the control group.

Tumour volumes and weights measured at the end of treatment are shown in Fig. 4. In comparison with the control group treated with scrambled siRNA-atelocollagen, the mean volume of tumours from the groups treated with 10 and $15 \mu \mathrm{M} F A B P 5$ siRNA-atelocollagen were significantly (Mann-Whitney $U$ test $\mathrm{p}=0.008$ and $\mathrm{p}=0.008$ ) reduced by 4.3- and 4.1-fold, respectively (Fig. 4a). When the control group was compared to groups treated with 1 or $5 \mu \mathrm{M}$ $F A B P 5$ siRNA-atelocollagen and $10 \mu \mathrm{M}$ siRNA in buffer, the average tumour volume was reduced, but not significantly (Mann-Whitney U test, $\mathrm{p}=0.15, \mathrm{p}=0.65, \mathrm{p}=0.2$ ), by $1.1-, 1.5$ and 1.2-fold, respectively.

The size of tumours was also assessed by weight measured at autopsy. The quantitative relationships amongst each group's mean tumour weight (Fig. 4b) were similar to those measured by tumour volume. In comparison with the control group treated with scrambled siRNA-atelocollagen, the mean tumour weight was significantly (Mann-Whitney $\mathrm{U}$ test $\mathrm{p}=0.02$ and $\mathrm{p}=0.02$ ) reduced by 3.4- and 3.8-fold in the groups treated with 10 and $15 \mu \mathrm{M} F A B P 5$ siRNA-atelocollagen, respectively.
Table I. C-FABP expression in tumours after different treatment. ${ }^{a}$

\begin{tabular}{lccccc}
\hline \multirow{2}{*}{ Treatment } & \multicolumn{5}{c}{ Staining intensities ${ }^{\mathrm{b}}$} \\
\cline { 2 - 6 } & & & + & ++ & ++ \\
\hline $10 \mu \mathrm{M}$ scrambled RNA-atelocollagen & 0 & 0 & 1 & 4 \\
$10 \mu \mathrm{M}$ siRNA in buffer & 1 & 3 & 1 & 0 \\
$1 \mu \mathrm{M}$ siRNA-atelocollagen & 0 & 0 & 5 & 0 \\
$5 \mu \mathrm{M}$ siRNA-atelocollagen & 0 & 4 & 1 & 0 \\
$10 \mu \mathrm{M}$ siRNA-atelocollagen & 3 & 2 & 0 & 0 \\
$15 \mu \mathrm{M}$ siRNA-atelocollagen & 3 & 2 & 0 & 0 \\
\hline
\end{tabular}

${ }^{a}$ Five animals in each group staining for C-FABP protein expression. ${ }^{a}$ Staining intensity classified as four categories: -, negative; +, weak; ++, moderate; +++, strong.

When compared with the control group, the average tumour weights from the groups treated with $1 \mu \mathrm{M}, 5 \mu \mathrm{M} F A B P 5$ siRNA-atelocollagen or $10 \mu \mathrm{M}$ siRNA in buffer were not significantly (Mann-Whitney $\mathrm{U}$ test, $\mathrm{p}=0.3, \mathrm{p}=0.6, \mathrm{p}=0.8$ ) different; either $<10 \%$, or $>10 \%$ and $20 \%$, respectively.

$C-F A B P$ expression in tumours. The immunohistochemical detection of C-FABP expression in tumours is summarized in Table I. Representative staining of tissues is illustrated in Fig. 5. In the group treated with scrambled siRNAatelocollagen, $80 \%$ of tumours stained strongly while $20 \%$ stained moderately (Fig. 5a). No significant difference $\left(\chi^{2}\right.$ test, $\mathrm{p}=0.1, \mathrm{p}=0.08)$ was detected between the staining intensities measured in the group treated with scrambled siRNA-atelocollagen and the groups treated with $1 \mu \mathrm{M} F A B P 5$ siRNA-atelocollagen and $10 \mu \mathrm{M} F A B P 5$ siRNA with buffer (Fig. 5b and f), respectively. In contrast, in groups treated, respectively, with 10 and $15 \mu \mathrm{M}$ siRNA-atelocollagen, $60 \%$ were unstained and $40 \%$ stained weakly positive in both groups. When compared with the group treated with scrambled siRNA-atelocollagen, the staining intensities detected in groups treated with 5, 10 and $15 \mu \mathrm{M}$ (Fig. 5c-e) FABP5 siRNA-atelocollagen, respectively, were significantly $\left(\chi^{2}\right.$ test, $\mathrm{p}=0.02, \mathrm{p}=0.01, \mathrm{p}=0.01$ ) reduced.

Western blotting of protein extracts from tissues of all six groups is shown in Fig. 6. Two tissues from each group have been shown as the representative of the group. Tissue treated with scrambled RNA was used as the reference and set as 1 and others were related to that. When the tissues treated with $10 \mu \mathrm{M}$ (nos. 7 and 8 ) and $15 \mu \mathrm{M}$ (nos. 9 and 10) FABP5 with atelocollagen compared to tissues treated with scrambled RNA (nos. 1 and 2), they show 1.3-1.6-fold and 1.7-2.3-fold reduction of C-FABP expression, respectively. Tissues treated with $1 \mu \mathrm{M}$ (nos. 3 and 4 ) and $5 \mu \mathrm{M}$ (nos. 3 and 4) were similar to control of a small amount of reduction. Tissues treated with siRNA alone show a 1.2-1.5 fold reduction compare to control. These data show $>50 \%$ reduction in CFABP protein expression in tumours treated with $15 \mu \mathrm{M}$ atelocollagen compare to the controls. 

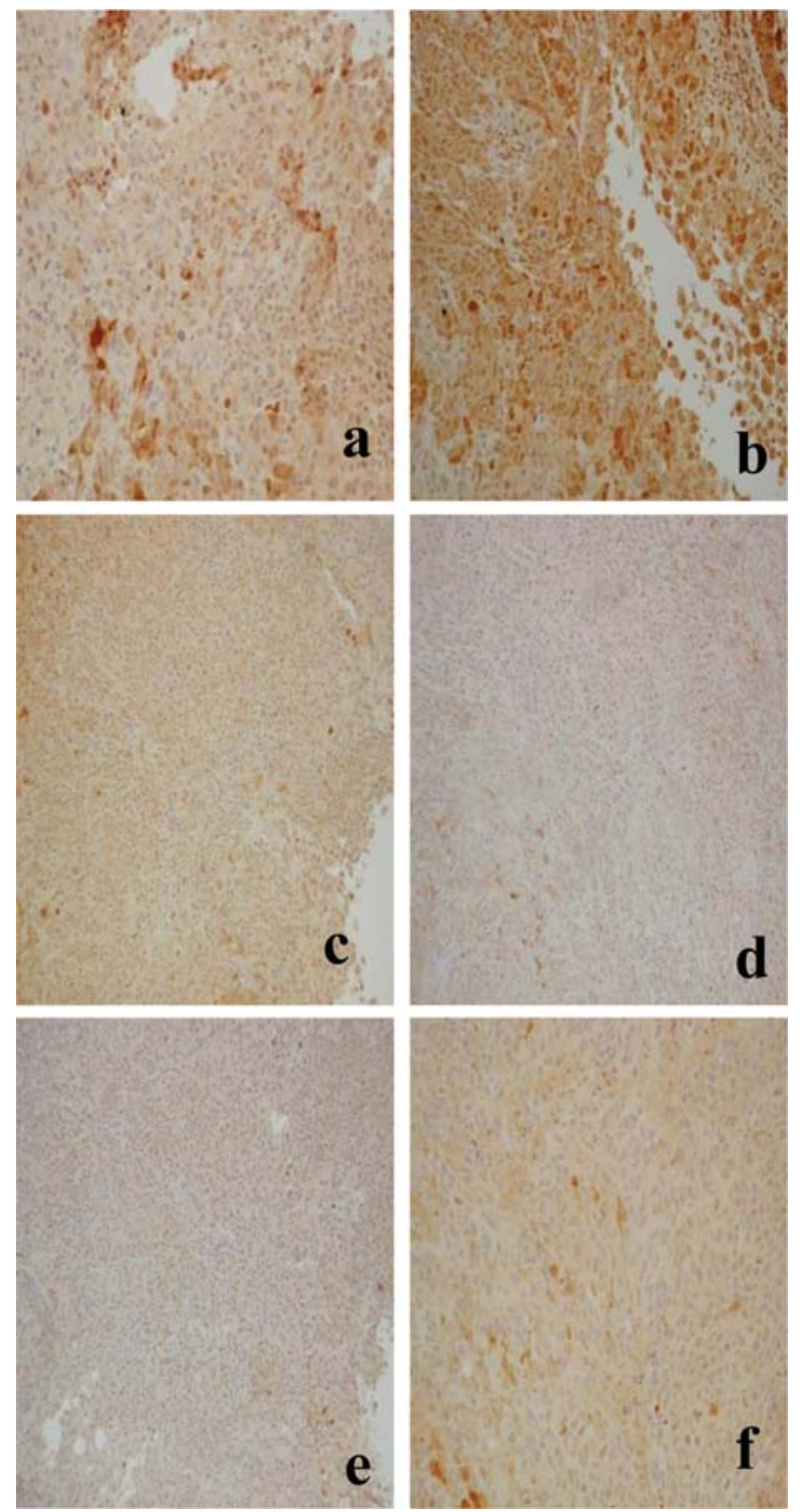

Figure 5. Immunohistochemical staining of C-FABP in tumours from 6 groups of mice after different treatments. (a) $10 \mu \mathrm{M}$ scrambled siRNA-atelocollagen (x250). (b) $1 \mu \mathrm{M}$ CFABP siRNA-atelocollagen (x250). (c) $5 \mu \mathrm{M}$ CFABP siRNA-atelocollagen (x100). (d) $10 \mu \mathrm{M}$ CFABP siRNA-atelocollagen (x100). (e) $15 \mu \mathrm{M}$ CFABP siRNA-atelocollagen (x250). (f) $10 \mu \mathrm{M}$ CFABP siRNA in buffer (x250).

\section{Discussion}

The findings of this study confirm the initial hypothesis and clearly demonstrate a dose-response relationship between siRNA molecules stabilised with atelocollagen delivered into the extracellular environment and the reduction in growth of prostate cancer cells. Previous studies performed as background to this work provided the evidence that stably-expressing siRNA targeting FABP5 within cancer cells effectively inhibits development of prostate cancer at the initiation stage $(3,10)$. However, it was not clear whether siRNA against FABP5 at the transcriptional level could effectively suppress the growth of tumours already established. Appropriate tools are required to effectively deliver siRNA molecules to tumours and to prevent their rapid degradation. Viral vectors (20-22) as means of delivering siRNA have been explored. Other a

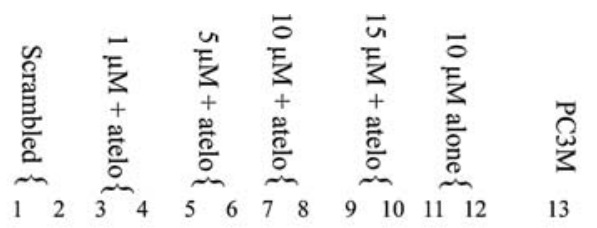

C-FABP

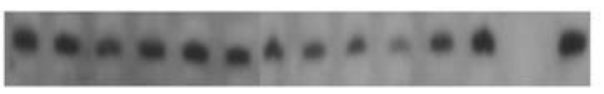

$\beta$-actin

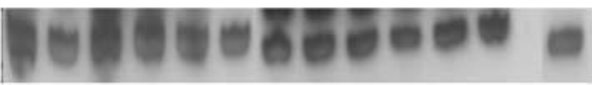

b

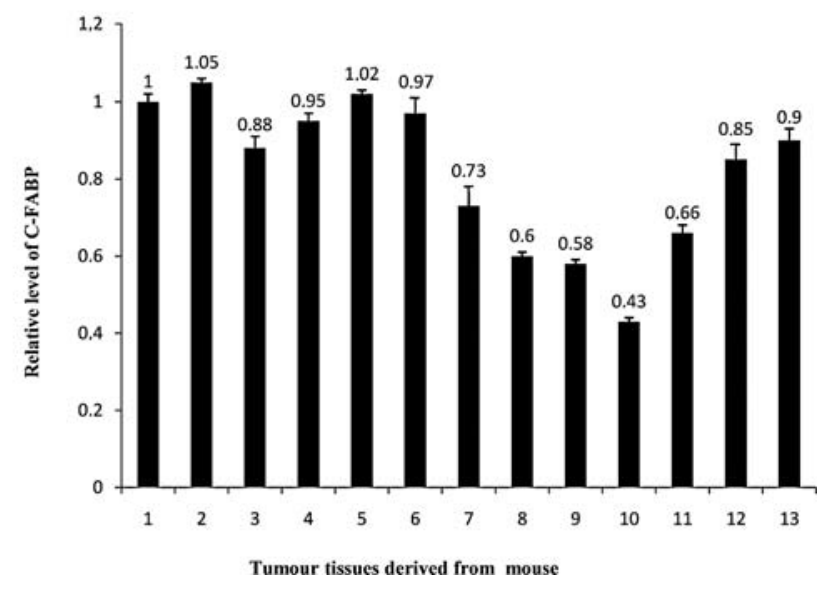

Figure 6. Levels of C-FABP expression in prostate tumours (physiological duplicates) from nude mice treated with; scrambled (nos. 1 and 2), $1 \mu \mathrm{M}$ (nos. 3 and 4), $5 \mu \mathrm{M}$ (nos. 5, 6), $10 \mu \mathrm{M}$ (nos. 7, 8), $15 \mu \mathrm{M}$ (nos. 9 and 10) siRNAs plus atelocollagen, siRNA alone (nos. 11, 12) and PC3M cells. (a) Western blot analysis of C-FABP expression in protein extracts from all 12 tissues and $\mathrm{PC} 3 \mathrm{M}$ cells. 3 -actin antibody was also incubated with the same blot to normalized the possible loading discrepancies. (b) Relative levels of C-FABP expressed in all tissue samples. The level of C-FABP expressed in tissues treated with scrambled siRNA was set at 1; C-FABP levels in the tissues treated with different dosage of siRNA were calculated relative to scrambled. The possible loading errors were corrected by normalizing values to $B$-actin, the result is shown as the mean $( \pm \mathrm{SE})$ of three separate measurements.

delivery systems, such as polyethylenimines (PEI) $(23,24)$, lipid-base siRNA delivery systems $(25,26)$ and the physical delivery of siRNA (27) were assessed previously. However, stabilising siRNA through atelocollagen was recently introduced as a more efficient approach by several groups that developed biomedical therapies to cancers of prostate (28), pancreas (29), and lung (30). Since siRNA molecules are negatively charged and atelocollagen is positively charged, electrostatic interactions between siRNA and atelocollagen make them to bind tightly and form complex with different physical shapes (31). Consequently, siRNA can remain stable inside the complex for about a week $(29,32)$. In addition, atelocollagen can change from a liquid state at low temperature to a solid state at body temperature thus increasing its protection of siRNA (9).

The detailed molecular mechanisms responsible for the biological effects of siRNA inside cancer cells are not completely clear, some controversial results having been reported $(7,33)$. Different siRNA sequences have different suppressing 
efficiencies on target genes as from 5 C-FABP sequences that we have tested, sequence 25 demonstrate the highest suppression.

Previously, it was discovered that targeting atelocollagenestabilised siRNA to VEGF in PC3 tumours effectively inhibited their growth (32) and that the optimal interval between each injection was 7 days $(29,32)$. Therefore, in the present study, the siRNA to FABP5 in atelocollagen was injected to the tumour mass on five occasions with 7-day intervals. The data showed that $C-F A B P$ siRNA delivered in buffer produced a suppression in tumour growth of $\sim 1$.2-fold (Fig. 4b), but this was not statistically significant. This finding indicated that although C-FABP siRNA in buffer at high dosage $(10 \mu \mathrm{M})$ was able to suppress tumour growth for a short time, initial suppression was reversed quickly and tumours continued to grow until the next injection. A RNA molecule can remain stable for $<24 \mathrm{~h}$ at body temperature. To maintain the treatment effect of siRNA without atelocollagen, it may be necessary to administer not only with high dose, but also with much higher frequencies (every $24 \mathrm{~h}$ ). Thus, application of atelocollagen can not only greatly save costs by reducing the amount of siRNA used and increase the treatment efficiency, but also avoids patients receiving frequent injections.

In this study, it was shown that suppression of tumour growth by siRNA-atelocollagen to FABP5 was related to the concentrations of siRNA administered. When the siRNA dose was too low $(1 \mu \mathrm{M})$, the complex did not suppress gene expression. When the dose was increased to $5 \mu \mathrm{M}$, the C-FABP protein level expressed by the tumour was significantly reduced, as detected by immunohistochemical analysis (Table I). However, this reduction was not sufficient to produce significant suppression of tumour growth.

Currently, the mechanism by which the gene FABP5 promotes tumourigenicity in prostate cancer cells is not completely clear and thus it is not understood how suppressing expression of C-FABP inhibits tumour growth. The previous study showed that $F A B P 5$ may facilitate malignant progression of prostate cancer cells by up-regulating the expression of VEGF (10), encoding a potent angiogenesis factor. Therefore, suppression of tumour growth caused by inhibiting FABP5 expression may be achieved, at least partially, by suppressing tumour angiogenesis (34). Blocking angiogenesis is efficient in preventing development of pre-malignant tumours but not very effective at suppressing expansion of tumours already established (35). It has been suggested that a good treatment effect could be achieved when tumours reached $50-60 \mathrm{~mm}^{3}$, since at this stage the vasculature of tumour tissues has not yet fully developed (32). In this study, siRNA to FABP5 at a concentration of $5 \mu \mathrm{M}$ was sufficiently high to produce significant reduction of $\mathrm{C}-\mathrm{FABP}$ in $\mathrm{PC} 3 \mathrm{M}$ cells, but not enough to produce suppression in tumour growth. A further increment of siRNA to $10 \mu \mathrm{M}$ not only produced a significant reduction of C-FABP expression but also a significant suppression of tumour growth. These results indicated that to achieve a treatment effect on already-developed tumours, a higher concentration of siRNA is requested to inhibit angiogeneic activity in addition to the amount of siRNA necessary to produce C-FABP suppression. Thus treatment, of the existing tumours may require a much higher siRNA dose than treatment of tumours at initial stage. Although significant suppression of tumour growth was achieved by FABP5-siRNA delivered with atelocollagen, none of the tumours were eradicated by this treatment. Thus, suppression of FABP5 expression in the growing $50-60 \mathrm{~mm}^{3}$ tumour masses can only inhibit, but not reverse, malignant progression.

In conclusion, the present study has confirmed the hypothesis that siRNA delivered in atelocollagen to inhibit expression of the gene FABP5 effectively suppresses the growth of established prostate carcinomas in vivo in a dosedependent manner. Furthermore, the study has demonstrated the effectiveness of delivering the siRNA molecules to the external environment of a tumour mass, and that for their effects, these molecules do not require delivery within the target cells. These findings strongly support the proposal that siRNA to the gene $F A B P 5$ is a potential adjunctive therapeutic tool in the treatment of localised prostate cancer, whether at a primary or secondary site.

\section{Acknowledgements}

We thank the North West Cancer Research Fund for the generous support of several research project grants.

\section{References}

1. Foster CS, Cornford P, Forsyth L, Djamgoz MB and Ke Y: The cellular and molecular basis of prostate cancer. BJU Int 83: 171-194, 1999.

2. Hughes C, Murphy A, Martin C, Sheils O and O'Leary J: Molecular pathology of prostate cancer. J Clin Pathol 58: 673-684, 2005.

3. Morgan EA, Forootan SS, Adamson J, et al: Expression of cutaneous fatty acid-binding protein $(\mathrm{C}-\mathrm{FABP})$ in prostate cancer: potential prognostic marker and target for tumourigenicitysuppression. Int J Oncol 32: 767-775, 2007.

4. Hannon GJ and Rossi JJ: Unlocking the potential of the human genome with RNA interference. Nature 431: 371-378, 2004.

5. Hanai K, Takeshita F, Honma K, et al: Atelocollagen-mediated systemic DDS for nucleic acid medicines. Ann NY Acad Sci 1082: 9-17, 2006.

6. Bumcrot D, Manoharan M, Koteliansky V and Sah DW: RNAi therapeutics: a potential new class of pharmaceutical drugs. Nat Chem Biol 2: 711-719, 2006.

7. Liu G, Wong-Staal F and Li QX: Development of new RNAi therapeutics. Histol Histopathol 22: 211-217, 2007.

8. Minakuchi Y, Takeshita F, Kosaka N, et al: Atelocollagenmediated synthetic small interfering RNA delivery for effective gene silencing in vitro and in vivo. Nucleic Acids Res 32: e109, 2004.

9. Ochiya T, Nagahara S, Sano A, Itoh H and Terada M: Biomaterials for gene delivery: atelocollagen-mediated controlled release of molecular medicines. Curr Gene Ther 1: 31-52, 2001.

10. Adamson J, Morgan E, Beesley C, et al: High-level expression of cutaneous fatty acid-binding protein in prostatic carcinomas and its effect on tumorigenicity. Oncogene 22: 2739-2749, 2003.

11. Kozlowski J, Fidler J, Campbell D, Xu Z, Kaighn M and Hart I: Metastatic behaviour of human tumour cell lines grown in the nude mouse. Cancer Res 44: 3522-3529, 1984.

12. Kaighn M, Lechner J, Narayan K and Jones L: Prostate carcinoma: tissue culture cell lines. Natl Cancer Inst Monogr 49: 17-21, 1978.

13. Ke Y, Jing C, Barraclough R, Smith P, Davies MP and Foster CS: Elevated expression of calcium-binding protein $\mathrm{p} 9 \mathrm{Ka}$ is associated with increasing malignant characteristics of rat prostate carcinoma cells. Int J Cancer 71: 832-837, 1997.

14. Forootan SS, Foster CS, Aachi VR, et al: Prognostic significance of osteopontin expression in human prostate cancer. Int J Cancer 118: 2255-2261, 2006. 
15. Forootan SS, Wong YC, Dodson A, et al: Increased Id-1 expression is significantly associated with poor survival of patients with prostate cancer. Hum Pathol 38: 1321-1329, 2007.

16. Janik P, Briand P and Hartmann NR: The effect of estroneprogesterone treatment on cell proliferation kinetics of hormonedependent GR mouse mammary tumours. Cancer Res 35: 3698-3704, 1975.

17. Foster CS, Gosden CM and Ke YQ: Primer: tissue fixation and preservation for optimal molecular analysis of urologic tissues. Nat Clin Pract 3: 268-278, 2006.

18. Kamalian L, Gosney JR, Forootan SS, et al: Increased expression of Id family proteins in small cell lung cancer and its prognostic significance. Clin Cancer Res 14: 2318-2325, 2008.

19. Zhang Y, Forootan SS, Liu D, et al: Increased expression of anterior gradient-2 is significantly associated with poor survival of prostate cancer patients. Prostate Cancer Prostatic Dis 10 293-300, 2007.

20. Xia H, Mao Q, Paulson HL and Davidson BL: siRNA-mediated gene silencing in vitro and in vivo. Nat Biotechnol 20: 1006-1010, 2002.

21. Hemann MT, Fridman JS, Zilfou JT, et al: An epi-allelic series of p53 hypomorphs created by stable RNAi produces distinct tumour phenotypes in vivo. Nat Genet 33: 396-400, 2003.

22. Rubinson DA, Dillon CP, Kwiatkowski AV, et al: A lentivirusbased system to functionally silence genes in primary mammalian cells, stem cells and transgenic mice by RNA interference. Nat Genet 33: 401-406, 2003.

23. Aigner A: Delivery systems for the direct application of siRNAs to induce RNA interference (RNAi) in vivo. J Biomed Biotechnol 2006: 1-15, 2006.

24. Aigner A: Gene silencing through RNA interference (RNAi) in vivo: strategies based on the direct application of siRNAs. J Biotechnol 124: 12-25, 2006

25. Obata Y, Suzuki D and Takeoka S: Evaluation of cationic assemblies constructed with amino acid based lipids for plasmid DNA delivery. Bioconjug Chem 19: 1055-1063, 2008.
26. Yoshizawa T, Hattori Y, Hakoshima M, Koga K and Maitani Y: Folate-linked lipid-based nanoparticles for synthetic siRNA delivery in KB tumour xenografts. Eur J Pharm Biopharm 70: 718-725, 2008.

27. Schiffelers RM, Xu J, Storm G, Woodle MC and Scaria PV: Effects of treatment with small interfering RNA on joint inflammation in mice with collagen-induced arthritis. Arthritis Rheum 52: 1314-1318, 2005.

28. Takeshita F, Minakuchi Y, Nagahara S, et al: Efficient delivery of small interfering RNA to bone-metastatic tumors by using atelocollagen in vivo. Proc Natl Acad Sci USA 102: 12177-12182, 2005.

29. Iwaki K, Shibata K, Ohta M, et al: A small interfering RNA targeting proteinase-activated receptor- 2 is effective in suppression of tumour growth in a Panc1 xenograft model. Int J Cancer 122: 658-663, 2008.

30. Kawata E, Ashihara E, Kimura S, et al: Administration of PLK-1 small interfering RNA with atelocollagen prevents the growth of liver metastases of lung cancer. Mol Cancer Ther 7: 2904-2912, 2008

31. Honma K, Ochiya T, Nagahara S, et al: Atelocollagen-based gene transfer in cells allows high-throughput screening of gene functions. Biochem Biophys Res Commun 289: 1075-1081, 2001.

32. Takei Y, Kadomatsu K, Yuzawa Y, Matsuo S and Muramatsu T: A small interfering RNA targeting vascular endothelial growth factor as cancer therapeutics. Cancer Res 64: 3365-3370, 2004.

33. Takeshita $\mathrm{F}$ and Ochiya T: Therapeutic potential of RNA interference against cancer. Cancer Sci 97: 689-696, 2006.

34. Leung DW, Cachianes G, Kuang WJ, Goeddel DV and Ferrara N: Vascular endothelial growth factor is a secreted angiogenic mitogen. Science 246: 1306-1309, 1989.

35. Bergers G, Song S, Meyer-Morse N, Bergsland E and Hanahan D: Benefits of targeting both pericytes and endothelial cells in the tumour vasculature with kinase inhibitors. J Clin Invest 111: 1287-1295, 2003 